\title{
A Note on Adaptive 2D-H Strings ${ }^{1}$
}

\author{
Ye-In Chang and Hsing-Yen Ann \\ Dept. of Applied Mathematics \\ National Sun Yat-Sen University \\ Kaohsiung, Taiwan \\ R.O.C. \\ \{E-mail: changyi@math.nsysu.edu.tw\} \\ \{Tel: 887-7-5252000 (ext. 3819)\} \\ $\{$ Fax: 886-7-5253809\}
}

\begin{abstract}
A picture is defined to be ambiguous if there exists more than one different reconstructed picture from its representation. In this paper, we first give an ambiguous case based on the adaptive 2D-H string representation [1]. Next, we show how to avoid the ambiguous cases.
\end{abstract}

(Keywords: 2D strings, 2D-H strings, adaptive 2D-H strings, image databases, symbolic pictures)

\footnotetext{
${ }^{1}$ This research was supported in part by the National Science Council of Republic of China under Grant No. NSC-87-2213-E-110-014.
} 


\section{Introduction}

In [3], Chang and Li has proposed 2D-H strings, which can be viewed as a combination of quadtrees [4] and 2D strings [2]. Using the 2D-H string, the hierarchical symbolic pictures can be represented efficiently in terms of space complexity. Although the $2 \mathrm{D}-\mathrm{H}$ string data structure has been proven to be an efficient approach to represent and to manipulate symbolic pictures, in [1], Chang and Lin has discovered some redundancies existing in those data representations. Therefore, they proposed another alternative, called adaptive 2D-H strings, for representing the relationships among the objects in an image.

In [1], Chang and Lin has presented an algorithm for converting symbolic pictures of any size into adaptive 2D-H strings. They show that their adaptive $2 \mathrm{D}-\mathrm{H}$ string can work well for many unbalanced non-square small pictures, which frequently exist in our real environment. However, based on Chang and Lin's procedure to construct the adaptive 2D-H string, ambiguous cases can occur, where a picture is defined to be ambiguous if there exists more than one different reconstructed picture from its representation. Therefore, in this paper, we first give an ambiguous case based on the adaptive $2 \mathrm{D}-\mathrm{H}$ string representation [1]. Next, we show how to avoid the ambiguous cases.

\section{An Ambiguous Case}

Take Figure 1 as an example, where picture $f_{1}$ and $f_{2}$ are two different pictures while they contain the same 4 symbols occupying 12 cells. The corresponding decomposition steps for pictures $f_{1}$ and $f_{2}$ are shown in Figure 2 and Figure 3, respectively.

Moreover, the corresponding adaptive 2D-H string representation for pictures $f_{1}$ and $f_{2}$ are as follows:

$$
\begin{aligned}
& \text { adaptive } 2 \mathrm{D}-\mathrm{H}\left(f_{1}\right) \\
& =b_{N} b_{S} s_{N} s_{S} \\
& =11 s_{N} s_{S} \\
& =11 \mathbf{1 0} s_{N N} s_{N S} \mathbf{1 1} s_{S N} s_{S S} \\
& =11 \mathbf{1 0} \underline{1001 A B} \mathbf{1 1} \underline{1000 C} \underline{01 D}
\end{aligned}
$$


adaptive $2 \mathrm{D}-\mathrm{H}\left(f_{2}\right)$

$=b_{W} b_{E} s_{W} s_{E}$

$=11 s_{W} s_{E}$

$=1110 s_{W W} s_{W E} \mathbf{1 1} s_{E W} s_{E E}$

$=11 \mathbf{1 0} \underline{1001 A B} \mathbf{1 1} \underline{1000 C} \underline{01 D}$

\section{The Revised Version of the Adaptive 2D-H Strings}

From the above example, we show that pictures represented in the adaptive 2D-H strings can be ambiguous. In this example, pictures $f_{1}$ and $f_{2}$ have the same corresponding quadtree as shown in Figure 4. To overcome this problem, we provide an answer. We can avoid the ambiguous case by adding the size information of a picture, say $m_{1} \times m_{2}$, at the end of the corresponding adaptive 2D-H string. The Reconstruct procedure presented in the Appendix shows how to reconstruct a picture based on the revised version of the adaptive 2D-H string without causing any ambiguous. In this Reconstruct procedure, we use the size information of a picture $f$, say $m \times n$, to guide us how to decompose the adaptive 2D-H string, just the same case as how the picture $f$ is segmented. In this way, obviously, when $m_{1} \neq m_{2}$ or $n_{1} \neq n_{2}$, two pictures $f_{1}$ (with size $m_{1} \times n_{1}$ ) and $f_{2}$ (with size $m_{2} \times n_{2}$ ) will be distinguished well even they have the same adaptive $2 \mathrm{D}-\mathrm{H}$ string representation.

\section{Conclusion}

The adaptive 2D-H string representation has been proposed to remove the redundancy existing in the 2D-H string representation. However, the concise representation of the adaptive 2D-H string can cause ambiguous cases. In this paper, we have shown such a case and have provided an answer to avoid the ambiguous case. 
$f_{1}$

\begin{tabular}{|l|l|}
\hline$A$ & \\
\hline & $B$ \\
\hline & \\
\hline$C$ & \\
\hline & \\
\hline & $D$ \\
\hline
\end{tabular}

(a) $f_{2}$

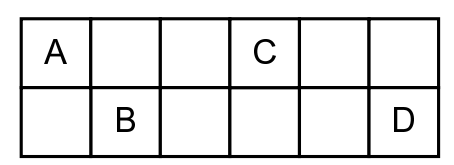

(b)

Figure 1: An example

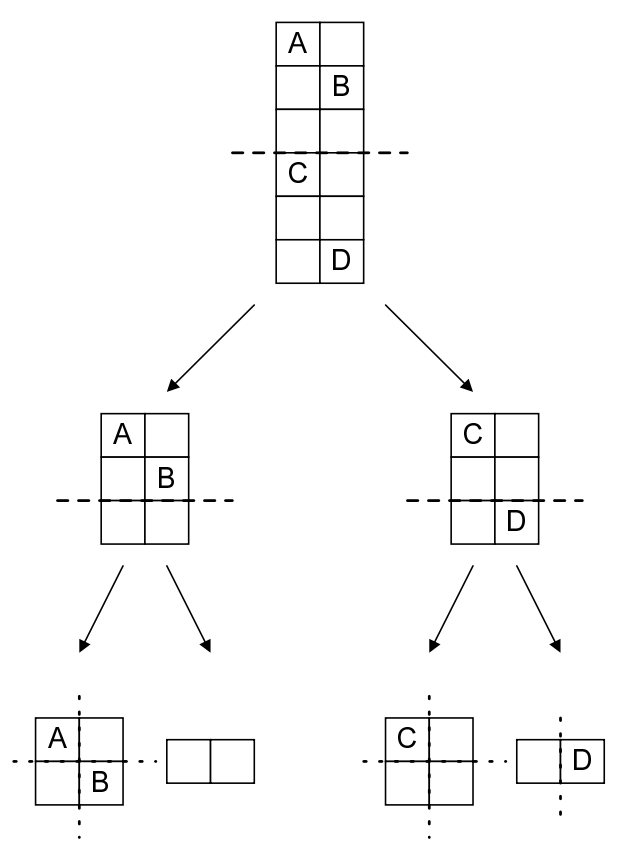

Figure 2: Decomposition steps for picture $f_{1}$ 


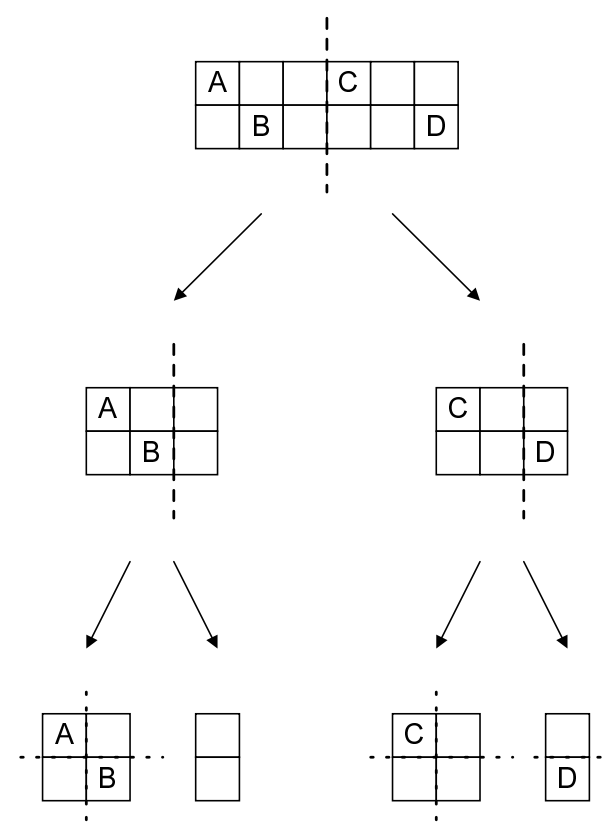

Figure 3: Decomposition steps for picture $f_{2}$

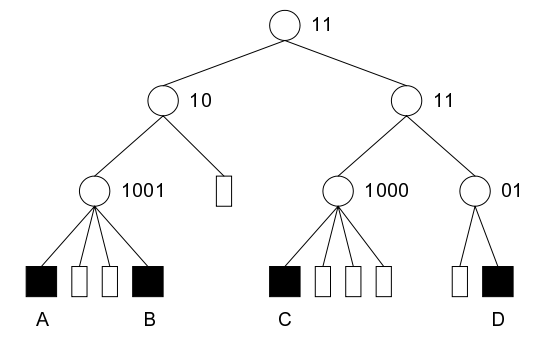

Figure 4: The quadtree of picture $f_{1}\left(f_{2}\right)$ 


\section{References}

[1] Chang, C. C. and Lin, D. C. (1996). A Spatial Data Representation: An Adaptive 2D-H String. Pattern Recognition Letters, 17 (2), 175-185.

[2] Chang, S. K., Shi, Q. Y. and Yan. C. W. (1987). Iconic Indexing by 2-D Strings, IEEE Trans. on Pattern Analysis and Machine Intelligence, PAMI-9 (3), 413-428.

[3] Chang, S. K. and Li, Y. (1988). Representation of Multi-Resolution Symbolic and Binary Pictures Using 2D-H Strings, Proc. IEEE Workshop on Languages for Automata, Maryland, 190-195.

[4] Samet, H. (1984). The Quadtree and Related Data Structure, ACM Comput. Surveys, 16 (2), 187-260.

[5] Samet, H. (1990). Applications of Spatial Data Structure, Addison-Wesley, Reading, MA. 


\section{Appendix}

\section{Procedure Reconstruct $(f, m, n)$}

Input: (1) the size of a symbolic picture $f, m, n$;

(2) a global variable $S$, the adaptive 2D-H string of $f$

Output: the symbolic picture $f$

1. IF $(\min (m, n)>2)$ THEN \% quadrant segmentation $\%$

2. BEGIN

3. $\quad$ set $f_{1}, f_{2}, f_{3}$ and $f_{4}$ to be NW, SW, NE and SE

4. quadrants subpictures of $f$, respectively

5 . $\quad \%$ let $S_{i}$ be the $i$ th bit of $S$ from the left side \%

6. $\quad$ FOR $i=1$ to 4

7. $\quad b_{i}:=S_{i}$

8. $S \leftarrow S$ shift left 4 bits

9. IF $\left(b_{1}=1\right)$ THEN

10. $\operatorname{Reconstruct}\left(f_{1},\lceil 1 / 2 m\rceil,\lceil 1 / 2 n\rceil\right) \quad \% \mathrm{NW} \%$

11. IF $\left(b_{2}=1\right)$ THEN

12. $\operatorname{Reconstruct}\left(f_{2},\lfloor 1 / 2 m\rfloor,\lceil 1 / 2 n\rceil\right) \quad \% \mathrm{SW} \%$

13. IF $\left(b_{3}=1\right)$ THEN

14. $\operatorname{Reconstruct}\left(f_{3},\lceil 1 / 2 m\rceil,\lfloor 1 / 2 n\rfloor\right) \quad \% \mathrm{NE} \%$

15. IF $\left(b_{4}=1\right)$ THEN

16. Reconstruct $\left(f_{4},\lfloor 1 / 2 m\rfloor,\lfloor 1 / 2 n\rfloor\right) \quad$ SE \%

17. END

18. ELSE IF $(m \leq 2$ and $n>2)$ THEN $\quad \%$ column segmentation $\%$

19. BEGIN

20. set $f_{1}$ and $f_{2}$ to be $\mathrm{W}$ and $\mathrm{E}$ quadrant subpictures of $f$

21. $\quad$ FOR $i=1$ to 2

22. $\quad b_{i}:=S_{i}$

23. $S \leftarrow S$ shift left 2 bits

24. IF $\left(b_{1}=1\right)$ THEN

25. $\quad \operatorname{Reconstruct}\left(f_{1}, m,\lceil 1 / 2 n\rceil\right) \quad \% \mathrm{~W} \%$

26. IF $\left(b_{2}=1\right)$ THEN

27. $\quad \operatorname{Reconstruct}\left(f_{2}, m,\lfloor 1 / 2 n\rfloor\right) \quad \% \mathrm{E} \%$

28. END

29. ELSE IF $(m>2$ and $n \leq 2)$ THEN $\quad \%$ row segmentation $\%$

30. BEGIN

31. set $f_{1}$ and $f_{2}$ to be $\mathrm{N}$ and $\mathrm{S}$ quadrant subpictures of $f$

32. $\quad$ FOR $i=1$ to 2

33. $\quad b_{i}:=S_{i}$

34. $S \leftarrow S$ shift left 2 bits

35. IF $\left(b_{1}=1\right)$ THEN

36. $\quad \operatorname{Reconstruct}\left(f_{1},\lceil 1 / 2 m\rceil, n\right) \quad \% \mathrm{~N} \%$

37. IF $\left(b_{2}=1\right)$ THEN

38. $\quad \operatorname{Reconstruct}\left(f_{2},\lfloor 1 / 2 m\rfloor, n\right) \quad \% \mathrm{~S} \%$ 


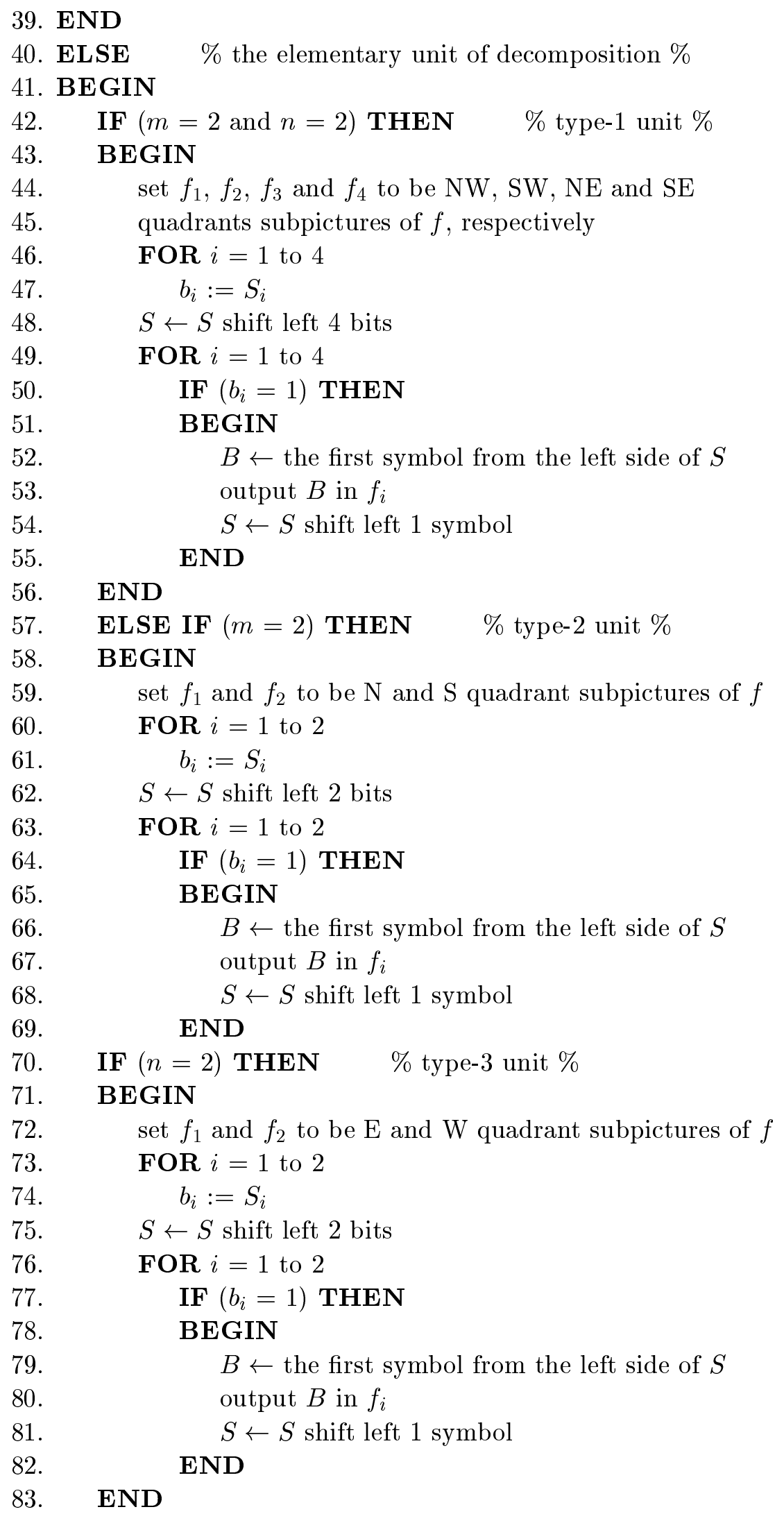


84. ELSE \% type- 4 unit \%

85. BEGIN

86. $\quad b_{1}:=S_{1}$

87. $\quad S \leftarrow$ shift left 1 bit

88. IF $\left(b_{i}=1\right)$ THEN

89. BEGIN

90. $\quad B \leftarrow$ the first symbol from the left side of $S$

91. $\quad$ output $B$ in $f_{i}$

92. $\quad S \leftarrow S$ shift left 1 symbol

93. END

94. END

95. END 